 \\ Revista Vol. 24(47) \\ Prolegómenos enero-junio - ISSN: 0121-182x · e-ISSN: 1909-7727 - pp. 117-132
}

\title{
El papel de las Fuerzas Militares estatales en la Cátedra de Paz y la seguridad: una mirada desde el departamento de Cundinamarca*
}

\author{
William Farfán Moreno ${ }^{a}$ - Luis Wuilliam Bravo Guerrab
}

\begin{abstract}
Resumen: uno de los objetivos del Acuerdo Final de paz de Colombia (2016) fue fomentar conocimientos y competencias sobre el territorio, la cultura, el contexto económico y social y la memoria histórica mediante la Cátedra de Paz. Cuando se plantean los temas relacionados con la memoria histórica y la paz en la escuela, surge la inquietud sobre el papel de las Fuerzas Militares (FFMM) estatales en la Cátedra de Paz. La pregunta surge porque, en la medida en que las FFMM, en el nivel institucional, son actores armados y, desde la subjetividad de sus miembros, algunos son víctimas del conflicto armado, también deben ser constructores de paz y aportar a la cátedra. Por tanto, el propósito del presente artículo es interpretar y analizar las percepciones que de las FFMm se tienen en la escuela, sobre la Cátedra de Paz, mediante el enfoque cualitativo, utilizando el método de análisis de contenido de los discursos de los participantes en los grupos focales (directivos, docentes y padres de familia de escuelas privadas y oficiales de Cundinamarca, Colombia), con el propósito de generar recomendaciones tanto para la cátedra como para la institución militar. Entre los resultados de la investigación, se encuentra que la percepción sobre la labor de las FFMM dentro de los procesos de enseñanza-aprendizaje en la Cátedra de Paz es que, a partir de la firma del Acuerdo Final y la implementación de la cátedra, se están repensando como actores, no solo como garantes del orden, sino como constructores de paz. Así también que el nuevo contexto obliga a resignificar el papel de las fFMm como actores para la paz.
\end{abstract}

* Artículo de investigación, resultado del proyecto "Esclarecimiento de la verdad histórica sobre la violencia estructural en Colombia, provocada al medioambiente y a las víctimas del conflicto. Aporte de las Fuerzas Militares en la reconstrucción del tejido social", del grupo de investigación Memoria Histórica, Construcción de Paz, Derechos Humanos, DICA y Justicia, categoría C por Colciencias (código coL0141423), vinculado al Centro de Investigación en Memoria Histórica Militar (CIMHM) y a la Maestría en Derechos Humanos, Derecho Internacional Humanitario y Derecho Internacional de los Conflictos Armados (DICA). Grupos adscritos y financiados por la Escuela Superior de Guerra "General Rafael Reyes Prieto", Colombia.

a Doctor en Ciencia Política de la Universidad de Belgrano, Buenos Aires; magíster en Filosofía de la Universidad de los Andes, y filósofo de la Universidad de La Salle. Investigador del Centro de Investigación en Memoria Histórica Militar de la Escuela Superior de Guerra General Rafael Reyes Prieto, Bogotá, Colombia. Correo electrónico: wifamo@yahoo.com ORCID: https://orcid.org/0000-0002-8198-2100

b Maestrando en la Maestría de Derechos Humanos y Derecho Internacional de los Conflictos Armados, Especialista seguridad y defensa nacional, Especialista en administración de recursos militares y Profesional en Ciencias Militares de la Escuela Superior de Guerra "General Rafael Reyes Prieto", Bogotá, Colombia. El presente artículo de investigación en coautoría es requisito de opción de grado para obtener el título de Magíster en Derechos Humanos y Derecho Internacional de los Conflictos Armados de la Escuela Superior de Guerra "General Rafael Reyes Prieto", Bogotá, Colombia. Correo electrónico: bravooperaciones@gmail.com 
Palabras clave: Cátedra de Paz; Fuerzas Militares estatales; paz; seguridad

Recibido: 04 de noviembre de 2020

Aceptado: 23 de febrero de 2021

Disponible en línea: 06 de agosto de 2021

Cómo citar: Farfán Moreno, W., \& Bravo Guerra, L. W. (2021). El papel de las Fuerzas Militares estatales en la Cátedra de Paz y la seguridad: una mirada desde el departamento de Cundinamarca. Prolegómenos, 24(47), 117-132. https:// doi.org/10.18359/prole.5423

\title{
The role of the State Military Forces in the Chair of Peace and security: a Look From the Department of Cundinamarca
}

\begin{abstract}
Final Peace Agreement in Colombia (2016) was to promote knowledge and skills about the territory, culture, the economic and social context and historical memory through the Chair of Peace. When issues related to historical memory and peace in the school are raised, concern arises about the role of the Military Forces in the chair. The question arises because, to the extent that the Military Forces, at the institutional level, are armed actors and, from the subjectivity of their members, some are victims of the armed conflict, they must also be peace builders and contribute to the Chair of Peace. Therefore, the purpose of this article is to interpret and analyze the perceptions of the Military Forces held in the school about the Chair of Peace through a qualitative approach using the method of content analysis of the participants' speeches in the focus groups (directors, teachers and parents of private and official schools of Cundinamarca, Colombia). The purpose is to generate recommendations for both, the Chair of Peace and the military institution.. Among the results of the research, it is found that the perception about the active work of the Military Forces within the teaching-learning processes in the Chair of Peace is that, from the signing of the Final Agreement and the implementation of the lecture, they are rethinking themselves as actors. Not only as guarantors of order but also as peace builders. Also, the new context forces us to resignify the role of the Military Forces as actors for peace.
\end{abstract}

Keywords: Chair of Peace; State Military Forces; peace; safety

\section{O papel das Forças Militares estatais na Cátedra de Paz e na segurança: um olhar a partir do departamento de Cundinamarca}

Resumo: um dos objetivos do acordo final de paz da Colômbia (2016) foi promover conhecimentos e competências sobre território, cultura, contexto econômico e social, e memória histórica mediante a Cátedra de Paz. Quando os temas relacionados com a memória histórica e a paz foram propostos na academia, surgiu o questionamento sobre o papel das Forças Militares (FMS) estatais na cátedra. A pergunta ocorre porque, à medida que as FMS, no nível institucional, são atores armados e, sob a subjetividade de seus membros, alguns são vítimas do conflito armado, também devem ser construtores de paz e contribuir para a cátedra. Portanto, o objetivo deste artigo é interpretar e analisar as percepções existentes das FMs na academia sobre a Cátedra de Paz, mediante a abordagem qualitativa, com a utilização do método de análise de conteúdo dos discursos dos participantes nos grupos focais (diretores, docentes e pais de famílias de escolas privadas e oficiais de Cundinamarca, Colômbia), a fim de gerar recomendações tanto para a Cátedra quanto para a instituição militar. Entre os resultados da pesquisa, encontra-se a percepção sobre o trabalho ativo das FMs dentro dos processos de ensino e aprendizagem na Cátedra de Paz é que, a partir da firma do acordo final e da implementação da cátedra, estão repensando-se como atores, não somente garantidores da ordem, mas também construtores da paz. Além disso, o novo contexto obriga a ressignificar o papel das FMs como atores para a paz.

Palavras-chave: Cátedra de Paz; Forças Militares estaduais; paz; segurança 


\section{Introducción}

En 2014, mediante la Ley 1732, el Gobierno nacional vio la necesidad de garantizar la creación y el fortalecimiento de una cultura de paz en Colombia, a través de la Cátedra de la Paz, en todas las instituciones educativas de preescolar, básica y media, como una asignatura independiente. Esta cátedra estuvo enmarcada en el fortalecimiento de la construcción de una paz "estable y duradera", de lo que luego fueron los compromisos de los Acuerdos de la Habana. De allí que, con el Decreto 1038 del 2015, el Ministerio de Educación Nacional (Mineducación) formalizara los lineamientos de la cátedra para fomentar el proceso de apropiación de conocimientos y competencias relacionados con el territorio, la cultura, el contexto económico y social, y la memoria histórica. Con ello, se buscaba reconstruir el tejido social, promover la prosperidad general y garantizar la efectividad los principios, derechos y deberes constitucionales.

Ese ministerio propuso doce puntos para la implementación de la cátedra, entre los que se encuentran la memoria histórica y reconciliación y la historia de los Acuerdos de Paz nacionales e internacionales. Estos dos temas se han convertido en la espada de Damocles de la implementación de la Cátedra de Paz. Este peligro deriva de que reconstruir la historia y la memoria histórica implica incluir las narrativas de los diferentes actores del conflicto armado, entre ellos, las Fuerzas Militares (FFMM) de Colombia.

$\mathrm{Si}$, en términos institucionales, las FFMM son actores armados, según contempla el Derecho Internacional Humanitario (DIH), y, desde la subjetividad de sus miembros, algunos de ellos se consideran víctimas del conflicto armado, entonces, como actores, ¿no deberían también ser constructores de paz desde la Cátedra de Paz? Precisamente, esta es la pregunta problemática que orienta este artículo. Para responderla, se planteó el objetivo de interpretar y analizar las percepciones sobre las FFMM que existen en la escuela, en torno a la Cátedra de Paz; mediante el enfoque cualitativo en el que se utiliza el análisis de contenido de los discursos de directivos, docentes de educación básica, secundaria y media fortalecida, y padres de familia de escuelas privadas y oficiales de Cundinamarca.

La recolección de los datos se hizo a través de tres grupos focales; y sus discursos se analizaron mediante las categorías de (1) paz, seguridad y (2) relación entre fFMm y Cátedra de Paz. Se utilizó la herramienta informática Atlas.ti para organizar y codificar la información. A partir del análisis y las interpretaciones, al final se realiza una serie de recomendaciones, a manera de conclusión, que podrían ser el horizonte de una nueva investigación.

\section{Marco contextual}

La Primera y Segunda Guerras Mundiales marcaron al mundo por las pérdidas humanas y violación a los Derechos Humanos. Después de la Guerra Fría, se pensó que los conflictos tendrían baja intensidad y las potencias mundiales generarían modelos y estrategias para reorganizar las sociedades en unas más dialogantes, con modelos pedagógicos que contribuyeran a una convivencia pacífica. Pese a ello, el siglo XxI se ha caracterizado por representar un sinnúmero de conflictos de alta intensidad, los cuales se han desarrollado a través de nuevas dinámicas en el empleo de la violencia.

Resultados obtenidos por estudios de la Organización Panamericana de la Salud (ops, 2016) muestran que la violencia afecta la salud de las personas, ya sea por acciones violentas como la discriminación, las amenazas con armas o sin ellas, la violencia sexual y las relaciones interpersonales violentas. En estos tipos de violencia, las mujeres y los niños son la mayoría de las víctimas. Además, un informe de la Organización Mundial de la Salud (oms, 2016), que incluyó 133 países, a cerca del $88 \%$ de la población mundial, determinó que

las tasas de respuesta por región abarcaron al $63 \%$ de la población en la región del Mediterráneo Oriental, el $70 \%$ en la región de África, el $83 \%$ en la región de Europa, el 88 \% en la región de las Américas y el $97 \%$ en las Regiones de Asia Sudoriental y del Pacífico Occidental. (p. 7)

Estas cifras demuestran que las nuevas formas de violencia son fenómenos presentes en todas las esferas de la sociedad, inclusive en los espacios 
educativos de muchos países. Los primeros estudios sobre violencia en centros escolares, realizados en Noruega y Suecia (1960-1980), evidencian el maltrato en las escuelas, la importancia del género, el curso y la posición de la víctima, el agresor y el testigo. En Australia, se efectuó una investigación en la que se estableció la diferencia entre maltrato maligno y no maligno, teniendo en cuenta la intencionalidad del agresor, así como algunas características de los actores: víctimas y agresores. En Finlandia e Inglaterra, se desarrollaron estudios basados en la influencia del género en el maltrato físico y psicológico en estudiantes, lo que permitió señalar algunas pautas para el desarrollo de estudios sobre género; también se evidenció la superioridad del maltrato de las escuelas de Inglaterra frente a las de Noruega (López de Mesa et al., 2013).

Colombia ha pasado por largos periodos de violencia, que incluyen diversos actores y fenómenos, tales como guerrillas, paramilitares, narcotraficantes, corrupción, extorsión, reclutamiento de menores, trata de personas, minería ilegal y violencia escolar, entre otras (Carvajal et al., 2012). A su vez, la violencia ha constituido un problema que ha afectado al país y ha permeado los diferentes contextos sociales, culturales y políticos. Es un fenómeno presente desde hace ya más de cincuenta años, vinculado con el conflicto armado y el narcotráfico, lo que ocasiona violencia social, expresada en actos como "ajustes de cuentas, venganzas y chantajes", presentes en la cotidianidad, y se refleja "en la seguridad personal y ciudadana y, por ende, en la convivencia en escenarios de la vida" (p. 386).

Por lo anterior, podemos aseverar que Colombia no ha sido ajena a estos fenómenos, los cuales han estado presentes antes, durante y después de la firma del Acuerdo Final entre el Estado y las antiguas Fuerzas Armadas Revolucionarios de Colombia Farc (Farc-EP), en 2016.

La Oficina del Alto Comisionado de las Naciones Unidas para los Refugiados (ACNUDH, 2020) observó un incremento en la respuesta militar a situaciones de violencia e inseguridad. Aunque existen protocolos, normativas y políticas públicas que regulan la participación del Ejército en situaciones relacionadas con la seguridad ciudadana, estos no fueron aplicados completamente en diferentes contextos de algunas zonas rurales de Arauca, Antioquia, Caquetá, Cauca, Córdoba, Cesar, Chocó, Meta, Nariño y Norte de Santander; incluso en centros urbanos como Convención, Medellín, Santa Marta y Valledupar. En estos lugares, el Ejército colombiano realizó operaciones antinarcóticos y de prevención y persecución de delitos, para los cuales el entrenamiento, equipamiento y naturaleza de las funciones militares son inapropiados. "Según datos de la Policía, en varios municipios de Arauca, Catatumbo, Norte del Cauca y Sur de Córdoba, se incrementó el número de homicidios, a pesar del aumento de la presencia militar" (ACNUDH, 2020, p. 11).

Del mismo modo, el departamento de Cundinamarca se convirtió en un corredor de los grupos al margen de la ley, por las características geográficas y por la cercanía de muchos de sus municipios con la capital y con los departamentos de Antioquia, Caldas, Huila, Meta, Boyacá, Casanare y Tolima. Las antiguas Farc-EP generaron terror, extorsionaron, desplazamiento, sometieron a la población civil y dominaron, por mucho tiempo, el territorio, organizadas por frentes para el control de la economía y de las finanzas:

El frente veinticinco (25) actuaba en la provincia del Sumapaz. El veintiocho (28), el treinta y ocho (38) y el cuarenta y dos (42) accionaban en los municipios del suroccidente, en la región del Tequendama y parte de las provincias del Bajo Magdalena y Sumapaz. Los frentes cincuenta y uno (51) ejercían influencia en el suroriente del departamento, en particular en la parte sur de la región Oriente. El cincuenta y dos (52) y el cincuenta y cinco (52) actuaban en la provincia del Sumapaz y desde el Meta y el frente cincuenta y tres (53) que presionaba la provincia de Medina (Secretaría de Planeación de Cundinamarca, 2019, p. 7)

Los efectos del conflicto armado en Cundinamarca son factores que han generado violencia dentro de las aulas de clase en instituciones educativas públicas y privadas. De acuerdo con la Comisión Económica para América Latina y el Caribe (Cepal, 2008),

el Programa para la gestión del conflicto escolar Hermes comenzó a funcionar en enero de 2001, por 
iniciativa de la Cámara de Comercio de Bogotá [...], dentro del marco de las acciones de responsabilidad social. Actualmente, funciona en 225 colegios de 19 localidades de Bogotá y en 10 municipios del departamento de Cundinamarca. Está concebido para atender grupos poblacionales entre 12 y 17 años que enfrentan situaciones de alta conflictividad tanto en el ámbito escolar como en el familiar y social. (p. 1)

A raíz de las situaciones de alta conflictividad en la escuela, el Gobierno nacional tomó la iniciativa de generar ambientes más pacíficos desde las aulas de clases. Por ello, promulgó la Ley 1732 (2104) de "Cátedra para la Paz". Por medio de esta ley, se reglamentó y estableció esta cátedra en todas las instituciones educativas del país, con el objetivo de educar para la paz. La cátedra está apoyada por el Ministerio de Educación Nacional y tiene lineamientos como los siguientes:

Fomentar el proceso de apropiación de conocimientos y competencias relacionados con el territorio, la cultura, el contexto económico y social y la memoria histórica, con el propósito de reconstruir el tejido social, promover la prosperidad general y garantizar la efectividad de los principios, derechos y deberes consagrados en la Constitución [...]. Contribuir al aprendizaje, la reflexión y al diálogo sobre los siguientes temas: cultura de la paz, educación para la paz y desarrollo sostenible. (Ley 1732, 2014, art. 2)

Otra de las formas y estrategias que el Estado colombiano estableció fue la Ley 1620 de 2013. Por medio del Decreto 1965 de 2013, con esta ley, el Ministerio de Educación Nacional promueve en los estudiantes el empoderamiento como sujetos activos de derechos, en cualquier contexto y en la responsabilidad de tomar decisiones para desarrollar su proyecto de vida. Esta norma es una estrategia para mitigar los conflictos escolares.

A pesar de los esfuerzos del Gobierno para mitigar los conflictos escolares en las instituciones educativas, en Cundinamarca siguen encontrándose actos de violencia en escuelas y colegios, como agresiones verbales entre pares, faltas de respeto entre estudiantes y maestros, amenazas con armas o sin ellas, conductas suicidas y baja motivación de grupos de estudiantes para permanecer en la escuela. Todos estos fenómenos pueden traer como consecuencia que las aulas se conviertan en escenarios de violencia y agresión a los Derechos $\mathrm{Hu}-$ manos y, a largo plazo, pueden atentar contra la seguridad en su sentido más amplio.

\section{Aproximaciones a la violencia escolar}

Los efectos de la violencia en el mundo, particularmente en Colombia, fueron una preocupación conjunta de los diferentes gobiernos, porque han transcendido a las aulas de clase de las diferentes instituciones educativas. De aquí que la violencia sea una preocupación que ha causado dolor, miedo, deserción, desplazamiento e inestabilidad política; por lo que tiene efectos en la vida escolar. La violencia es una de las infortunadas respuestas a la resolución de los conflictos y, de aquí, que pueda señalarse que el conflicto se presenta en cualquier contexto donde coexistan grupos de personas. El conflicto existe cuando las personas perciben que las actividades desarrolladas para la consecución de sus objetivos son incompatibles con las actividades y deseos de otras personas y grupos.

Por el contrario, para Jares (2002), el conflicto debe ser visto como un valor que debe generar debate y servir de base para la crítica pedagógica. Para Galtung (2003), el conflicto es crisis y oportunidad, porque es un hecho natural y estructural permanente en el ser humano. Para el sociólogo noruego, el conflicto no se soluciona, sino que se transforma; e implica una experiencia vital holística, como dimensión estructural de las relaciones.

Ahora bien, los conflictos que se resuelven por la vía violenta tienen un asidero también en los contextos escolares. Para Cabrales (2017), por ejemplo, la violencia escolar tiene factores de riesgo familiar, problemáticas macroestructurales, violencia entre pares y problemática de drogas. Como sustenta Boggino (2005), en las últimas décadas, en Latinoamérica, la escuela y el aula han sido sacudidas por diversos atravesamientos, fruto de políticas de exclusión, por nuevas pautas culturales propias de la posmodernidad y por las profundas crisis socioeconómicas. 
Como sostienen Blaya et al. (2006), las causas de la violencia entre los jóvenes en las instituciones educativas podrían estar en el ambiente escolar, entendido como las relaciones entre pares, maestros y estudiantes, maestros y padres de familia y entre maestros (Fernández, 1994). Las relaciones interpersonales y los roles que se establecen en las escuelas son un factor determinante en las formas de comportamientos de cada miembro de la comunidad educativa.

El Fondo de las Naciones Unidas para la Infancia (Unicef, 2011) realizó estudios en varios países de América Latina y del Caribe para determinar las causas que generan violencia y maltrato en niños, niñas y adolescentes, en espacios escolares; lo que conlleva a fenómenos como el castigo físico, el abuso sexual, el maltrato emocional, los golpes físicos de maestros a estudiantes y entre pares, y burlas y humillaciones entre compañeros. Dentro de los resultados principales de este estudio, se plantean las llamadas dos revoluciones: (1) la revolución del modelo tradicional del trato entre los adultos y los niños en las escuelas; y (2) la revolución del conocimiento y del acceso a la información, mediante las nuevas tecnologías de la comunicación, que podrían generar nuevos riesgos de violencia, así como nuevos mecanismos de prevención. En este contexto, el estudio enfatiza en la promoción de una educación basada en los Derechos Humanos y el reconocimiento de los niños como sujeto de derechos.

El Comité de los Derechos de los Niños, en su Observación General número 13, sostiene que el derecho a la educación en un medio para el desarrollo satisfactorio de otros derechos. De aquí surge la importancia y la necesidad de que se promueva y se garantice en los Estados miembro de la onu. Puede verse, de manera implícita, que aplicar la normativa que se dispone en la Observación General número 13 ayudaría a promover espacios de convivencia pacífica, puesto que dentro de sus propósitos y objetivos está promover la dignidad de la personalidad humana, la participación en sociedades libres y la comprensión de la diversidad étnica, cultural y religiosa entre todos.

En términos de la violencia escolar, se destaca el numeral 41 en el que se hace hincapié en la necesidad de no emplear ningún método violento como disciplina escolar para los niños, niñas y adolescentes, ya que ello iría contra el principio rector fundamental de los Derechos Humanos: la dignidad humana. Asimismo, estableció que la defensa del derecho del niño contra toda forma de violencia es responsabilidad de todos y su principal componente son las medidas educativas:

El Estudio Mundial realizado por la Unicef reconoció que la violencia contra los niños, niñas y adolescentes en las escuelas y centros de aprendizaje se perpetra a través de formas de violencia física o psicológica, que pueden ser realizadas por profesores, personal de las instituciones educativas - con o sin aprobación de las autoridades gubernamentales- y, en algunos casos, por otros niños. (Unicef, 2011, p. 19)

Los efectos de la violencia escolar son múltiples. Debido a un entorno en el que los niños, niñas y jóvenes, sin reglas ni normas en la familia, trasladan sus conductas agresivas a la escuela; sin tener control de sus emociones para resolver los problemas en cualquier contexto.

Para Chaux (2011), el problema de la violencia escolar requiere de múltiples perspectivas para resolver un problema tan complejo. Según este autor, "hoy sabemos, también, que la agresión que sufren muchos estudiantes puede tener consecuencias académicas y psicológicas graves en el corto y largo plazo" (p. 76). En otras palabras, la violencia escolar genera interrupciones académicas e impide los procesos socializantes en la convivencia entre pares, maestros y estudiantes y las relaciones padres y maestros.

Países como México, Bolivia, Chile, Perú, Paraguay y Colombia han publicado leyes sobre convivencia, seguridad y violencia escolar. De acuerdo con Rivera (2012), las escuelas mexicanas y la legislación sobre la convivencia, seguridad y la violencia escolar, pretenden generar las condiciones indispensables para la construcción y fortalecimiento de una convivencia escolar sin violencia. En Colombia, la Ley 1620 de 2013 creó el Sistema Nacional de Convivencia Escolar y Formación para el ejercicio de los Derechos Humanos, la educación para la sexualidad y la prevención y mitigación de la violencia escolar. 


\section{Cátedra de la Paz en Colombia}

La Constitución de 1991 promulgó: "La paz como un derecho y un deber de obligatorio cumplimiento" (art. 22); a lo que se suma: "El ejercicio de los derechos y libertades reconocidos en la constitución implica responsabilidades entre ellas, propende al logro y al mantenimiento de la paz" (art. 95). Estos principios implican que Colombia se adhiere a los tratados internacionales, según los cuales, la educación debe tener un enfoque de Derechos Humanos y paz. Además, con los artículos constitucionales mencionados, el Estado considera a la paz un derecho humano superior, así como un requisito importante para el ejercicio de todos los derechos y deberes restantes de los ciudadanos.

La Ley 115 de 1994 definió elementos como la participación, la paz, los Derechos Humanos y la democracia, definida en los objetivos de la educación. Asimismo, el plan decenal 2006-2016 (Mineducación, 2007), conocido como el "Pacto social por el derecho a la educación”, estableció en sus fines la educación en y para la paz, y la convivencia; con sus macroobjetivos de inclusión, diversidad, identidad y equidad. A partir de estos elementos, se buscaba que se crearan y propongan políticas públicas para el fomento de la convivencia, el desarrollo sostenible, diversidad social, económica y cultural del país, desde las aulas.

Finalmente, el Estado colombiano buscó involucrar a las instituciones educativas para fomentar una cultura de paz en el país, en el contexto de las negociaciones entre el Estado y las Farc-EP, en La Habana. Ello, no solo con el fin del conflicto armado, sino también para la construcción y las garantías de los territorios desde la educación para la paz y los Derechos Humanos, mediante la Ley 1732 de 2014, conocida como "Cátedra para la Paz".

Por su parte, el Ministerio de Educación Nacional, con su Decreto 1038 del 2015 diseñó los lineamientos para articular aquella ley con las mallas curriculares y planes de estudio de todas las instituciones educativas públicas y privadas. Tales lineamientos son de obligatorio cumplimiento y tienen el objetivo de contribuir al aprendizaje, la reflexión y el diálogo en torno a la cultura de paz.

\section{Abordaje conceptual}

\section{Concepciones sobre la paz}

Para el propósito y la delimitación del presente abordaje conceptual, se expone inicialmente la idea de paz, desde el análisis del politólogo y filósofo Norberto Bobbio (1981). Posteriormente, se desarrolla este desde el principal representante de las llamadas "investigaciones para la paz" (peace research) de Johan Galtung (1969). Luego, se desarrolla uno de los últimos debates sobre la paz bajo los conceptos de (1) paz humanitaria y (2) guerra humanitaria para, finalmente, abordar la relación entre paz y seguridad.

Bobbio (1981) realiza un acercamiento a la definición de paz desde la peace research, donde solo puede proveerse una definición de paz en relación y estrecha conexión con la definición de guerra. Define la guerra mediante tres connotaciones vinculantes entre sí: (1) la guerra es un conflicto, (2) entre grupos políticos independientes, (3) cuya solución se confía a la violencia organizada.

Por conflicto, Bobbio entiende la situación en la que las necesidades o intereses de un individuo o colectivo se satisfacen, sacrificando las necesidades de otro individuo o grupo. Por grupos políticos entiende, a su vez, a grupos organizados que buscan mantener u obtener el máximo de poder posible. Quien detenta el poder dispone del monopolio de la fuerza física. Por último, entiende la violencia organizada como (1) "el uso de la fuerza física", (2) "intencionalmente dirigida a lograr el efecto deseado por el sujeto activo"; y (3) "no consentida por parte del sujeto pasivo” (p. 163).

Así pues, desde su definición de guerra, Bobbio (1981) define la paz de la siguiente manera:

La paz, entendida como no-guerra, puede definirse como el estado en que se encuentran grupos políticos entre los cuales no existe una relación de conflicto, caracterizada por el ejercicio de una violencia duradera y organizada. De esto se deriva que (1) dos grupos políticos pueden estar en conflicto entre sí sin estar en guerra, ya que el estado de paz no excluye el conflicto que se realiza mediante el ejercicio de la violencia duradera y organizada; (2) dos grupos 
políticos no deben considerarse en estado de guerra si en sus relaciones se verifican casos de violencia esporádica, como lo son, por ejemplo, los incidentes fronterizos. (p. 164)

Desde esta perspectiva, es posible identificar dos tipos de definiciones sobre la paz: una paz negativa y una paz positiva, que no son las mismas que empleó Galtung. La primera no es otra cosa que su definición en su acepción puramente semántica. Es decir, la paz como ausencia de guerra. En cuanto a la paz positiva, se comprende desde el lenguaje técnico jurídico. Es una acepción específica, con la cual la paz se entiende no tanto como la ausencia de guerra, sino como el fin o la solución jurídicamente regulada de una guerra. Es positiva en tanto que, además de rechazar la definición negativa, caracteriza a la paz como un valor positivo, que permite buscar la justicia, esto es, un estado de cosas deseable (Bobbio, 1981).

Por otra parte, el filósofo y sociólogo francés Aron, según Bobbio (1981), distingue tres tipos de paz: de potencia, de impotencia y de satisfacción. La primera se subdivide en tres: paz de equilibrio, de hegemonía y de imperio. Esta subdivisión corresponde con las situaciones de equilibrio o desequilibrio en que se encuentren los grupos políticos entre sí. Si hay una condición de igualdad es una paz de equilibrio; si se encuentran en una condición en la que hay mayor ponderación de un grupo político sobre otros (como el caso de EE. UU. con respecto a los demás Estados de la región, según Bobbio) es una paz hegemónica; finalmente, si hay una condición de dominio ejercido por la fuerza, como la Pax Romana, es una paz de imperio.

Por su parte, la paz de impotencia de Aron, como explica Bobbio (1981), es aquella caracterizada por la era atómica o la Guerra Fría. Es la paz de suma cero, desde la teoría de juegos, que no es otra cosa que la tesis de la Destrucción Masiva Asegurada. Es la paz de un mero equilibrio de fuerzas oponentes.

Ahora bien, la paz por satisfacción es la más interesante para Bobbio (1981). De ahí que parta de ella para analizar los conceptos y formas de pacifismo: "La paz de satisfacción tiene lugar cuando en un grupo de estados ninguno tiene pretensiones territoriales o de otro tipo frente a los demás, y sus relaciones están basadas sobre la confianza recíproca” (p. 176).

El concepto de paz por satisfacción también es analizado por Rawls en Law of Peoples (1999), fundamentada en dos ideas. La primera es que las instituciones políticas y sociales pueden ser revisadas y reformadas con el propósito de hacer felices a los pueblos; la segunda es lo que Montesquieu entendía por costumbres moderadas y se refiere a que una sociedad comercial tiende a fomentar virtudes para incentivar el comercio, y el comercio, a su vez, tiende a la paz. Al juntar estas dos ideas, se infiere que los pueblos democráticos dedicados al comercio no librarían la guerra entre sí, pues la satisfacción de las necesidades básicas se logra más fácilmente a través del comercio que de la guerra; y esto es compatible con los pueblos democráticos.

Otras concepciones sobre la paz se encuentran en Galtung (1969), quien distingue, por un lado, entre la paz negativa entendida como la ausencia de violencia física o personal y, por otro, la paz "positiva", entendida como la ausencia de violencia estructural, represión e injusticia:

Nos referimos al tipo de violencia donde hay un actor que comete la violencia de forma tanto directa como personal, y la violencia donde no hay tal actor como estructural o indirecta [...]. Puede que no haya ninguna persona que dañe directamente a otra en la estructura. La violencia está incorporada en la estructura y se muestra como un poder desigual y, por consiguiente, como oportunidades desiguales en la vida. ${ }^{1}$ (p. 171; traducción propia)

De acuerdo con la distinción entre las violencias personal y estructural, Galtung (1969) identifica dos tipos de paz, a saber, la paz negativa y la paz positiva:

1 "We shall refer to the type of violence where there is an actor that commits the violence as personal or direct, and to violence where there is no such actor as structural or indirect [...]. There may not be any person who directly harms another person in the structure. The violence is built into the structure and shows up as unequal power and consequently as unequal life chances” (Galtung, 1969, p. 171). 
Con la distinción entre la violencia personal y estructural como base, la violencia se convierte en dos facetas y, así, lo hace la paz, concebida como la ausencia de violencia. Un concepto extendido de violencia conduce a un concepto de paz. Tal como una moneda, tiene dos caras, siendo una sola cara solo un aspecto de la moneda, no la moneda completa, la paz también tiene dos caras; la ausencia de violencia personal y la ausencia de violencia estructural. Nos referiremos a ellas respectivamente como paz negativa y paz positiva. ${ }^{2}$ (p. 183; traducción propia)

En A Mini Theory of Peace, Galtung (2007) argumenta: "La paz no es una propiedad de una sola parte, sino una propiedad de la relación entre las partes"3 (p. 71; traducción propia). De este modo, distingue entre relaciones negativas (no armoniosas), indiferentes y positivas (armoniosas). En las primeras, lo que es malo para uno es bueno para el otro; en las indiferentes, no hay preocupación por el otro; por último, en las relaciones positivas, lo que es bueno para uno lo es también para el otro. Dichas relaciones, afirma Galtung (2007), coinciden en el mundo y se manifiestan como paz negativa y paz positiva.

De este modo, Galtung define la paz negativa tanto como la ausencia de violencia y un alto al fuego, como la ausencia de las relaciones negativas (no armoniosas) e indiferentes. Por otra parte, define la paz positiva como la presencia de armonía y la ausencia de todo aquello que tenga el potencial de obstruir el bienestar.

2 "With the distinction between personal and structural violence as basic, violence becomes two-sided, and so does peace, conceived of as the absence of violence. An extended concept of violence leads to an extended concept of peace. Just as a coin has two sides, one side alone being only one aspect of the coin, not the complete coin, peace also has two sides: absence of personal violence, and absence of structural violence. We shall refer to them as negative peace and positive peace, respectively” (Galtung, 1969, p. 183).

3 "Peace is not a property of one party alone, but a property of the relation between parties" (Galtung, 2007, p. 71).
Otro debate sobre la paz, contemporáneo al de Bobbio y Galtung, se ha dado en el contexto de la filosofía política y las relaciones internacionales, bajo el contraste de dos conceptos novedosos e interdependientes: (1) guerra humanitaria y (2) paz humanitaria. Este debate es recogido por la filósofa Teresa Santiago en La guerra humanitaria, pasado y presente de una controversia filosófica (2013). Santiago inicia explicando los postulados de quienes defienden la guerra humanitaria —entendida como intervencionismo-; luego, los de quienes la rechazan; finalmente, expone un nivel intermedio, que es donde nace el concepto de paz humanitaria.

Los defensores de la guerra humanitaria sustentan las intervenciones militares con el argumento de la defensa de los Derechos Humanos y la protección de las víctimas en Estados donde tales derechos son violados y vulnerados sistemáticamente. Por encima de la soberanía de los Estados como valor político, están la protección y garantía de los derechos fundamentales de los individuos. De aquí que esta línea defensora de la guerra humanitaria defienda la tesis de la doctrina de la guerra justa.

La contraparte de los defensores de la guerra humanitaria es la llamada línea crítica, cuyos autores tienen en común el rechazo total, en todas sus formas, del intervencionismo humanitario. Entre los autores importantes de esta corriente se encuentran Noam Chomsky (2002), Danilo Zolo (2006), Alain Badiou (2003) y Anthony Burke (2005). En general, los autores de esta línea cuestionan y se niegan a aceptar las nociones de causa justa y guerra humanitaria de los defensores del humanitarismo. Santiago (2013) afirma que "quienes participan de ese amplio debate contribuyen a la justificación filosófica de una política internacional que pretende avasallar cualquier obstáculo en el camino de la gran potencia y sus aliados" (p. 149).

Entre quienes defienden la guerra humanitaria y quienes la rechazan, se encuentra un grupo de teóricos de la guerra y las relaciones internacionales que asumen una posición intermedia: la paz humanitaria. Esta tendencia teórica parte de dos presupuestos. Por un lado, asumen una precariedad del Derecho Internacional, incapaz de imponer sanciones a las naciones violadoras de los Derechos Humanos y pusilánime ante el poder 
de las potencias mundiales; por otro, rechazan la doctrina de la guerra justa, debido a que invoca el recurso bélico y sostienen que la guerra moderna con sus medios y recursos de destrucción masiva no pueden ser utilizadas por instituciones internacionales que afirman proteger los Derechos $\mathrm{Hu}$ manos (Santiago, 2013).

La propuesta central radica, desde la postura de Mary Kaldor (2007), en que quien debe tomar el liderazgo para afrontar y revertir la crisis de violaciones de los Derechos Humanos no es la comunidad internacional sino la sociedad civil. Según Santiago (2013), comentando a Kaldor, esta posición "incorpora a la posible solución del problema a la sociedad civil, porque de ahí debe surgir la presión para cambiar las condiciones materiales que hacen posible el fenómeno recurrente de los crímenes contra la humanidad" (p. 163).

\section{Relación paz y seguridad}

Waever (2009) desarrolló un análisis conceptual de paz y su relación con la seguridad desde un rastreo histórico, tomando la metodología de la Historia conceptual de Koselleck ${ }^{4}$, desde el Imperio romano hasta antes de la finalización de la Segunda Guerra Mundial (1945). Posteriormente, hizo lo propio tanto en el contexto de la Guerra Fría, como en la época posterior a ella.

Con respecto a la primera parte de su estudio, entre sus conclusiones se encuentra que, durante la Edad Media, el concepto de paz fue más importante

4 Bödeker (2009) sintetiza este método historiográfico de la siguiente forma: "La historia de los conceptos en sentido estricto, según Reinhart Koselleck, es una tarea historiográfica: consiste en la historia de la formación de conceptos, sus estructuras de experiencia y contextos de acontecimientos en la longue durée. La historia de los conceptos o historia conceptual (Begriffsgeschichte) interroga -en tanto historia de los significados de términos escogidos- acerca del alcance social de los conceptos en sus respectivos contextos históricos; tematiza la potencia vinculante, acuñadora, caracterizadora de los conceptos en grupos políticos y sociales; y analiza el cambio estructural en el ámbito de la época, de la sociedad y de la política en tanto este es analizable como cambio en la experiencia, en las expectativas y en la teoría” (p. 132). que el mismo concepto de seguridad. Este último no fue más que un concepto político, que se relacionó con las concepciones de paz y justicia. No obstante, durante la configuración de los Estados modernos y en búsqueda de la "paz nacional", la seguridad adquirió un rasgo sobresaliente, hasta el punto de llegar a definir los horizontes de la paz.

En la primera mitad del siglo $\mathrm{xx}$, la seguridad adquirió la forma de seguridad colectiva y, luego, de seguridad nacional. Mientras que la paz, en el contexto de la guerra, se convirtió en un concepto ilusorio o poco adecuado para los problemas políticos tan profundos que se vivieron (Waever, 2009). Así, la seguridad se transformó en el concepto central desde 1940. A partir del análisis conceptual e histórico, Waever (2009) concluyó que

ambos términos, paz y seguridad, tienen historias mucho más ricas que las que apreciamos en el paquete moderno de sus acepciones. Cada una tiene relación con otras esferas y significados, aunque, en ciertos contextos, hayan sido conectadas correlativamente. Sin embargo, durante la Guerra Fría fue diferente, debido a que ambos conceptos rivalizaron hasta un grado extraordinario. (p. 83)

Tal rivalidad entre los conceptos de paz y seguridad se debió a la bipolaridad dada durante la Guerra Fría. Waever (2009) explica este fenómeno desde tres elementos: (1) la posición de la onU; (2) la bipolaridad Oriente-Occidente; y (3) la década de 1980. En cuanto al punto 1, la perspectiva de la onu, la Carta de las Naciones Unidas (1999) contempla el concepto de paz como "paz universal", en el sentido de evitar guerras (mundiales); también en el sentido amplio de promover los Derechos Humanos, el desarrollo económico y las relaciones cordiales entre Estados. En cuanto a seguridad, utiliza el concepto de "seguridad internacional" y no propiamente seguridad nacional. Sin embargo, advierte que la seguridad nacional solo puede realizarse como seguridad internacional. En este sentido, la seguridad internacional no busca más que aportar seguridad nacional de manera pacífica.

En cuanto a la polaridad Oriente-Occidente, Waever (2009) sostiene que "la seguridad asumió el lugar de la paz en el sentido tradicional de prevención de la guerra" (p. 86). Mientras en Oriente 
se utilizaba más el concepto de paz y no el de seguridad debido a que las fuentes marxistas-leninistas no lo utilizaban; Occidente fue más propenso a usar más el concepto de seguridad. La razón se centra, sostiene Waever (2009), en que Occidente tenía serios problemas con la idea de una paz eterna desarrollada en el contexto de las Guerras Mundiales y los totalitarismos. Occidente no confiaba, tampoco, en la lectura historicista y absoluta de la ideología del liberalismo que lograra la paz eterna.

Finalmente, respecto al tercer elemento, la década de 1980 fue la época en el que en Europa occidental emergieron los llamados movimientos pacifistas que tenían como finalidad evitar la propagación de armas nucleares. También surgieron intelectuales que apoyaban dichos movimientos y se encargaron de las llamadas "investigaciones para la paz". Estos movimientos sociales e intelectuales "trataron de adoptar el término seguridad que antes había sido utilizado solo por el poder dominante" (Waever, 2009, p. 88).

No obstante, los intelectuales ortodoxos de las investigaciones para la paz polemizaron con esta postura al sostener que la seguridad era el problema y no la solución del sistema internacional, toda vez que su enfoque no era otra cosa que retomar la lógica de la Guerra Fría (carrera armamentista). Así, para los ortodoxos, sus investigaciones e interpretaciones sobre el Sistema Internacional de 1980 se centraban en el concepto de paz, no en el de seguridad.

Frente a la postura ortodoxa surge también la de los "reformadores", quienes "en contraste, trataron de acercarse al poder dominante al retomar y redefinir el término seguridad" (Waever, 2009, p. 89). En cuanto a este grupo, conviene tener en cuenta también que "mucho de lo que se ha logrado en cuanto a redefinir el concepto de seguridad viene de esta tendencia" y que, entonces,

se introdujeron nuevos conceptos como "seguridad común" y "alianzas de seguridad" (así como defensa no-ofensiva), y la seguridad como tal se amplió más allá de sus restricciones militares. Algunos radicales también se dieron ocasionalmente a la tarea de redefinir el concepto de seguridad (¿quizá parcialmente como una reacción a los reformadores?) y esto conllevó a la más extrema ampliación del concepto de seguridad en la historia (Galtung, Øberg). (p. 89)

Mientras los ortodoxos emprendieron una lucha académica por posicionar el concepto de paz, los reformadores intentaron redefinir el concepto de seguridad. Esto último llevó a plantear un debate entre quienes sustentaban una concepción amplia de la seguridad con los de la concepción limitada al campo militar. Como expresa Waever (2009), "surgió un dualismo lleno de tensiones, en un movimiento popular que hablaba de paz y de sus intelectuales que empleaban el término seguridad reformada" (p. 90).

Por lo anterior, es claro que se necesitaba un punto intermedio entre el extremismo de la paz (investigadores para la paz), que soslayaban la seguridad, y el extremismo del poder (el realismo de las relaciones internacionales y los estudios estratégicos). Dicho intermedio, como sustenta Waever (2009), sería Barry Buzan, quien en un ensayo publicado en el Journal of Peace Research argumentó que la seguridad sería el punto de consenso entre estos extremos. Nació así el Instituto de Investigación para la Paz de Copenhague, cuyo objetivo principal fue repensar la seguridad con base en la defensa no-ofensiva de Europa.

Durante el periodo posterior a la Guerra Fría, la paz reapareció como un concepto central del mundo occidental: "Cuando la tarea del Oeste cambió de luchar en una Guerra Fría a construir un 'nuevo orden internacional', de pronto recordó que, de hecho, tenía una visión de paz de largo plazo como democracia y liberalismo" (Waever, 2009, p. 89). Así, los distintos discursos presidenciales de EE. UU. en la posguerra se convirtieron en los abanderados de este proyecto occidental:

- Bush padre, en su discurso del "Nuevo orden mundial” tras la finalización de la Guerra del Golfo Pérsico en 1991, afirmó: "Una paz duradera debe ser nuestra misión".

- Bill Clinton sostuvo su teoría de la "paz democrática".

- Bush hijo, en su discurso de posesión, después de los eventos del Once de Septiembre de 2001, afirmó: "La paz y la libertad prevalecerán" (Waever, 2009). 
No obstante, ello no significa la desaparición del concepto de seguridad en la pos-Guerra Fría: el concepto ha venido interactuando con otros desarrollos teóricos como la sociedad de riesgo y la seguridad humana. El primero es un tipo de seguridad respecto a las grandes amenazas del siglo XXI, como el terrorismo y el cambio climático; el segundo apunta a los temas de desarrollo y de la centralidad de los individuos más que de los Estados (Waever, 2009).

\section{Metodología}

El método de investigación cualitativa empleado fue el análisis de contenido. La razón principal por la que se empleó este enfoque es que permite analizar los fenómenos o hechos sociales desde las concepciones o cogniciones de los actores sin desarraigarse de la subjetividad de los investigadores. Los investigadores tienen la función de interpretar los marcos de representación sobre los fenómenos o hechos sociales que tienen la característica de ser variables:

En la aproximación cualitativa hay una variedad de concepciones o marcos de interpretación, que guardan un común denominador: todo individuo, grupo o sistema social tiene una manera única de ver el mundo y entender situaciones y eventos, la cual se construye por el inconsciente, lo transmitido por otros y por la experiencia, y mediante la investigación, debemos tratar de comprenderla en su contexto. (Hernández et al., 2014, p. 9)

En este orden, la investigación empleó la técnica del análisis de contenido de datos recogidos a través de tres grupos focales, distribuidos en docentes y directivos de básica primaria, docentes y directivos de básica secundaria y media fortalecida; y padres de familia de instituciones privadas y oficiales de Cundinamarca. El análisis de contenido permite analizar e interpretar los marcos de representación de los participantes mediante los discursos sobre un fenómeno social (Bardin, 1986) que, en esta investigación, son sobre la Cátedra de Paz y el papel que tendrían las FFMM en el proceso de enseñanza-aprendizaje.

Como sostiene López (2002), el análisis de contenido en concordancia con el enfoque cualitativo permite dar objetividad y subjetividad a la investigación:

En tanto que esfuerzo de interpretación, el análisis de contenido se mueve entre dos polos: el del rigor de la objetividad y el de la fecundidad de la subjetividad. Disculpa y acredita en el Investigador esa atracción por lo oculto, lo latente, lo no aparente, lo potencial inédito, lo no dicho, encerrado en todo mensaje. (p. 173)

La objetividad y la subjetividad son la capacidad del investigador para interpretar los marcos de representación presentes en los discursos, mediante categorías de análisis que, para este trabajo de investigación, fueron: paz, relación paz y seguridad, y relación Cátedra de Paz y fFMm Las dos primeras categorías trabajadas y deducidas de teorías sobre la paz y la seguridad (abordaje conceptual). La subjetividad permite enriquecer y aportar el conocimiento sobre el fenómeno social estudiado. Por último, la herramienta informática que permitió categorizar y sistematizar los discursos (corpus) de los participantes en los tres grupos focales fue Atlas.ti.

\section{Resultados}

El trabajo de los grupos focales fue desarrollado con docentes, directivos y padres de familia, donde se trabajó el concepto de paz, junto con, por un lado, su relación con la seguridad y, por otro, el papel de las FFMM en los procesos de construcción de paz desde la educación. El trabajo con estos grupos focales arrojó resultados importantes que pueden ser insumos para revisar y hacer recomendaciones sobre la malla curricular de la Cátedra de la paz en las instituciones educativas. Aquí, se presenta el análisis por cada una de las tres categorías trabajadas en los grupos focales: paz; paz y seguridad; y fFMm y Cátedra de Paz.

\section{Paz}

Según lo analizado en los grupos focales, a la luz del abordaje conceptual, pudo interpretarse que la paz, desde los actores, adquirió varias connotaciones: la paz humanitaria; la paz como valor político; y la paz como un estado de no conflicto y ausencia de violencia estructural. Los participantes, 
actores fundamentales en el proceso de enseñanzaaprendizaje y testigos de los diferentes momentos de conflictividad violenta en las escuelas y en el departamento, consideran que la necesidad de construir paz, de modo que la sociedad civil logre empoderarse de los procesos.

Ven en la educación la mejor forma para que todos los actores de la escuela - estudiantes, docentes, directivos, padres de familia- se empoderen en los procesos de construcción de la paz, no solamente en sus colegios y departamento, sino en la construcción de los Acuerdos de la Habana, como veedores de su implementación. Consideran que la paz humanitaria como empoderamiento se hace desde la escuela con la enseñanza-aprendizaje de los procesos sociohistóricos que llevaron al conflicto armado en Colombia; con miras a superarlos y aportar a la construcción de escenarios pacíficos en la escuela.

En cuanto valor político, consideran que no puede verse como un concepto abstracto, sino como un valor que debe aprenderse desde el hogar y reafirmarse en la escuela, con el fin de incidir en la trasformación de una sociedad justa, participativa y democrática. En este sentido, el adjetivo político se entiende no como un fenómeno adherente a una ideología o partido, sino como el escenario que garantiza todas las condiciones necesarias y suficientes para que cada individuo pueda vivir y lograr su florecimiento humano.

En efecto, la idea de la paz como valor político es consecuente con la paz como derecho de obligatorio cumplimiento (artículo constitucional 22). Cumple la función de derecho, cuando, en estado de guerra, todos los ciudadanos pueden exigirla constitucionalmente; y como deber, en cuanto, mientras haya guerra, el Estado está en la obligación de buscarla.

La concepción de la paz como valor político es diferente a la de una paz como valor supremo. Para los participantes es claro que la paz es un medio que permite alcanzar otro tipo de valores superior como la justicia, la participación y la libertad. Desde esta percepción de la paz, no como un fin, sino como un medio se puede evidenciar la compatibilidad con los lineamientos curriculares de la cátedra de la paz propuesto por el Ministerio de Educación.

\section{Relación paz y seguridad}

Los participantes, en general, tienen una idea ortodoxa de la seguridad centrada en el "orden". Una de las razones por las que tienen esta idea tradicional de seguridad tiene que ver con las experiencias que han vivido. Por ejemplo, hay presencia de estudiantes reinsertados o hijos de reinsertados de grupos armados ilegales. De ahí que asocien la seguridad solamente con el elemento de defensa para mantener el orden. Esta percepción de seguridad se asocia con el concepto de paz negativa de Galtung. Es claro que, ante un conflicto armado interno, con las características específicas de Cundinamarca, el primer paso hacia la construcción de la paz es buscar los mecanismos necesarios para la ausencia de la guerra. Aquí conviene precisar que fue en el escenario del Acuerdo Final cuando surgió la Cátedra de Paz y que ese acuerdo contemplaba la dejación de armas y el cese bilateral del fuego entre las partes (Farc-EP y Gobierno de Colombia).

Por otra parte, cuando los participantes hablan de la paz como la necesidad de superar los problemas estructurales paz positiva de Galtung, no lo relacionan con la idea de seguridad. Ello deriva, precisamente, de que tienen una percepción tradicional de ella. Lo que se colige de esto es que no hay una percepción de una seguridad amplia, tema en el que debe trabajarse.

De este modo, cuando se habla de paz negativa, la asocian con las Fuerzas Militares, porque son las encargadas de mantener el orden con el monopolio legítimo de la fuerza, cuando hay conflicto armado. Sin embargo, desconocen la labor que podrían tener en la paz positiva, al desconocer otras concepciones de seguridad multidimensional, como la que se propone con la seguridad humana, que busca, en efecto, fortalecer los temas del desarrollo humano y su centralidad es la gente más que en los Estados.

\section{La Cátedra de la Paz y las FFMM}

Inicialmente hay un análisis centrado en la Cátedra de la paz desde la perspectiva de los docentes quienes, como se mencionó, están involucrados en 
estos procesos de enseñanza-aprendizaje en las escuelas de Cundinamarca.

Entre los docentes, existe una idea errónea generalizada según la cual "son adoctrinadores ideológicos", pero su función es formar el pensamiento crítico de los estudiantes para que puedan asumir con criterio propio lo que ha significado el conflicto armado en Colombia, la memoria histórica y la construcción de paz. Esta idea de adoctrinamiento evoca la polarización política e historiográfica sobre la reconstrucción de la memoria histórica y los orígenes y desarrollo del conflicto armado. En otras palabras, si existe una idea de que los docentes adoctrinan es porque existen posturas y críticos que no están de acuerdo con las narrativas y los contenidos de lo que se enseña en la Cátedra de Paz.

Lo anterior plantea que la enseñanza-aprendizaje sobre la cátedra sugiere la necesidad de revisar, por un lado, las razones por las cuales existe una estigmatización hacia los docentes; por otro, el currículo, que debe ser el resultado de un método constructivista y horizontal en que participen varios actores.

Tanto docentes como padres de familia ven una desconexión entre la Cátedra de la Paz con el núcleo familiar de los estudiantes. En otras palabras, no ven un alcance de la cátedra en los espacios de la familia, con actividades y didácticas que las incluyan. Los participantes coincidieron en que la construcción de paz, como proponen los lineamientos de Mineducación, es un proceso que, si bien se hace en la escuela, debe tener impacto en la familia, ya que consideran que la violencia estructural también tiene lugar en los núcleos familiares y se reproduce en otros ecosistemas sociales.

En cuanto a la percepción que, en el contexto de la Cátedra de la Paz, se tiene de las Fuerzas Militares existen tres posturas que pueden categorizarse en la percepción institucional (garantes del orden y actores armados); y la percepción desde la subjetividad (como víctimas del conflicto armado). En la percepción institucional, por una parte, perciben a las FFMM como la institución del Estado que, en medio del conflicto armado, garantizan el orden público mediante la agresión y el monopolio legítimo de la fuerza. Aquí, los participantes no mencionan la idea de seguridad cuando se mencionan las FFMM.

Por otra parte, las perciben como actores armados del conflicto, lo cual tiene sentido, pues así son considerados jurídicamente desde el DIH. Sin embargo, una interpretación generalizada de los "actores" en un conflicto armado no internacional puede llevar a que ubiquen a las FFMM en el estatus de los grupos armados organizados (GAO), distanciándolos de los principios del DIH. Las Fuerzas Armadas estatales son consideradas "combatientes", es decir, personas con derecho a participar directamente en hostilidades; mientras que los GAO son un grupo de personas con una estructura de poder organizada, que portan armas y participan en hostilidades como actor no estatal, no sujeta al DIH (Melzer, 2019).

Desde la percepción de la subjetividad, entendiendo al sujeto militar no desarraigado de su contexto individual, social e histórico, dentro del conflicto armado, los participantes identifican a las fFMm como víctimas. Deviene, en este aspecto, un desdoblamiento entre ser "actores armados" y "víctimas" al mismo tiempo.

Así pues, surge una pregunta: ¿cómo es posible que los actores armados no sean los victimarios y sí las víctimas? Precisamente, cuando se reviste a los militares de su subjetividad e individualidad, sin desconocer su condición de combatientes legítimos, no significa desconocer que algunos de sus integrantes fueron víctimas cuando los GAO violaron principios del DIH, relacionados con la parte enemiga del conflicto.

Finalmente, la percepción sobre la labor activa de las FFMm dentro de los procesos de enseñanza-aprendizaje en la Cátedra de Paz es que ellas, a partir de la firma del Acuerdo Final y la implementación de la cátedra, están repensándose como actores, no solo como garantes del orden, sino como constructores de paz. En otras palabras, han visto positivamente las acciones para vincularse con la escuela para investigar, analizar y proponer estrategias de cultura de paz.

Repensarse en este escenario obliga a resignificarse y resignificar el papel de las FFMM no como actores para la guerra, sino actores para la paz. Esta interpretación surgió cuando un docente 
participante afirmó que, cuando ha escuchado a sus estudiantes decir que desean ser militares o policías, lo hacen pensando en la guerra y no para hacer la paz, en el sentido positivo de Galtung.

\section{Recomendaciones: a manera de conclusiones}

A partir de estas percepciones tanto del quehacer docente como del papel de las FFMM, en el contexto de la Cátedra de la Paz, con las categorías de paz y seguridad, el cruce de estas percepciones permite plantear algunas de las recomendaciones:

1. Teniendo en cuenta el abordaje conceptual y el análisis de los grupos focales, la Cátedra de $\mathrm{Paz}$ debe pensarse en la paz, sin desconocer la importancia de la seguridad en su sentido amplio. Concretamente, en la categoría de Educación para la Paz "Memoria histórica y reconciliación", cuyos temas de trabajo son la memoria histórica y la historia de los Acuerdos de Paz nacionales e internacionales, según propone Mineducación (Chaux y Velásquez, 2017), se recomienda trabajar las diferentes concepciones de paz - como se expuso - sin desligarlo de la idea de seguridad, en el que el centro de la securitización sean los individuos y no los Estados.

2. Al querer incluir las narrativas de las FFMM estatales en la construcción de la memoria histórica y la construcción de la paz con una seguridad en sentido amplio, es importante que el currículo de Cátedra de la Paz sea un proceso constructivista y multiperspectivista, en el que también se incluya la voz de las FFMM, en tanto actores y víctimas del conflicto.

3. En congruencia con el punto anterior, de acuerdo con los análisis de los resultados, es importante que la Cátedra de Paz tenga un proceso de enseñanza-aprendizaje circular, cuya centralidad sean los estudiantes y sus periferias sean la familia, la escuela y la comunidad de sus región o departamento.

4. Desde las fғмm, en su calidad de institución estatal, se requiere realizar capacitaciones sobre Cátedra de Paz para que, dentro de sus funciones multimisionales, puedan incorporarse en el proceso, como una forma de hacer parte de la construcción de la paz estable y duradera.

5. Del mismo modo, se recomienda desarrollar actividades de Cátedra de Paz, a través de coordinaciones interinstitucionales que vinculen entidades gubernamentales y no gubernamentales. Con el desarrollo de estas actividades, se busca resaltar la importancia de las FFMm y su rol legítimo en la construcción de Paz y reconstrucción de tejido social.

\section{Referencias}

Asamblea General de Naciones Unidas (1999, 06 de octubre). Declaración y programa de acción sobre una cultura de paz (A RES/53/24). Autor. http://undocs.org/ sp/A/RES/53/243

Badiou, A. (2003). La ética. Herder.

Bardin, L. (1986). El análisis de contenido. Akal.

Blaya, C., Debarbieux, E., Rey, R. y Ortega, R. (2006). Clima y violencia escolar. Un estudio comparativo entre España y Francia. Revista de Educación, 339, 293-315.

Bobbio, N. (1981). El problema de la guerra y las vías de la paz. Gedisa.

Bödeker, H. (2009). Sobre el perfil metodológico de la historia conceptual. Temas, problemas, perspectivas. Historia y Grafía, 32, 131-168.

Boggino, N. (2005). Convivir, aprender y enseñar en el aula. Homo Sapiens.

Burke, A. (2005). Against the new internationalism. Ethics and International Affair Journal, 19(2), 73-89.

Cabrales, V. (2017). Problemáticas de convivencia escolar en las instituciones: Análisis desde la pedagogía (Tesis doctoral, Universidad del Norte).

Comisión Económica para América Latina y el Caribe Cepal. (2008). Programa para la gestión del conflicto escolar. Cámara de Comercio de Bogotá.

Chaux, E. (2011). Múltiples perspectivas sobre un problema complejo: violencia escolar. Psykhe, 78-79.

Chuax, E. y Velásquez, A. (2017). Orientaciones generales para la implementación de la cátedra de la paz en los establecimientos educativos de preescolar, básica y media de Colombia. Mineducación.

Chomsky, N. (2002). El nuevo humanitarismo militar. Siglo Veintiuno. 
Congreso de Colombia (1994, 8 de febrero). Ley general de educación [Ley 115]. Diario Oficial, No. 41214 . https:// bit.ly/3fncvCi

Congreso de Colombia (2014, 01 de septiembre). Por la cual se establece la Cátedra de la Paz en todas las instituciones educativas del país [Ley 1732]. Diario Oficial, No. 49261. https://bit.ly/3ePdQ5Y

Congreso de Colombia (2013, 15 de marzo). Sistema nacional de convivencia escolar y formación para el ejercicio de los Derechos Humanos, la educación para la sexualidad y la prevención y mitigación de la violencia escolar [Ley 1620]. Diario Oficial, No. 48733. https:// bit.ly/3ojPIeX

Constitución política de Colombia [Const.] (1991). 2. ${ }^{\mathrm{a}}$ Ed. Legis.

Fondo de las Naciones Unidas para la Infancia -Unicef. (2011). Violencia escolar en América Latina y el Caribe. Superficie y fondo. Panamá: Autor.

Galtung, J. (1969). Violence, peace, and peace research. Journal of Peace Research, 6(3), 167-191.

Galtung, J. (2003). Violencia cultural. Gernika Gogoratuz. Primera edición de 1980.

Galtung, J. (2007, 04 de enero). A Mini Theory of Peace. https://bit.ly/3htEsey

Hernández, R., Fernández, C. y Baptista, P. (2014). Metodología de la investigación. 6. ${ }^{\mathrm{a}}$ Ed. Mc Graw-Hill.

Jares, X. R. (2002). Aprender a convivir. Revista Interuniversitaria de Formación del Profesorado, 44, 79-92.

Kaldor, M. (2007). El poder y la fuerza. Tusquets.

López de Mesa, C., Carvajal, C. A., Urrea, P. N. y Soto, M. F. (2014). Factores asociados a la convivencia escolar en adolescentes. Educación y Educadores, 16(3), 383-410.

López, F. (2002). El análisis de contenido como método de investigación. Revista de Educación, 4, 167-179.
Carvajal, C., Urrea, P. y Soto, M. (2012). La convivencia escolar en adolescentes de cinco municipios de sabana centro (Tesis de maestría, Universidad de la Sabana). Repositorio institucional Unisabana. https://bit.ly/2RdBiRA

Melzer, N. (2019). Derecho Internacional Humanitario. Una introducción general. Comité Internacional de la Cruz Roja.

Ministerio de Educación Nacional -Mineducación (2017). Plan nacional decenal de educación 2006-2016. Compendio general. "Pacto social por la educación". https:// bit.ly/3tOPA8v

Organización Mundial de la Salud -oms. (2016). Informe sobre la situación mundial de la violencia 2014. Autor.

Presidencia de la República (2015, 25 mayo). Por el cual se reglamenta la Cátedra de la Paz [Decreto 1038]. Diario Oficial, No. 49522. https://bit.ly/3yfTJ8D

Rawls, J. (1999). The law of peoples; with the idea of public reason revisited. Harvard University Press.

Rivera, Z. (2012). Concepciones e implicaciones de tres leyes antibullying en México. Diálogos sobre educación. Temas actuales en investigación educativa, 3(4), 1-21.

Santiago, T. (2013). La guerra humanitaria. Pasado y presente de una controversia filosófica. Gedisa.

Secretaría de Planeación de Cundinamarca (2019). Plan de Desarrollo Departamento de Cundinamarca. Autor.

Waever, O. (2009). Paz y seguridad: dos conceptos en evolución y su relación cambiante. En: U. Oswald y H. Günter (Comp. y Eds.). Reconceptualizar la seguridad en el siglo XXI (pp. 71-99). Centro Regional de Investigaciones Multidisciplinarias. Disponible en https://bit. ly/3uUZHdi

Zolo, D. (2006). La justicia de los vencedores. De Nuremberg a Bagdad. Trotta. 